\title{
Associations Between High Levels of High-Density Lipoprotein Cholesterol and the Presence and Severity of Coronary Artery Disease in Patients Who Have Undergone Coronary Computed Tomography Angiography
}

\author{
Kohei Tashiro $^{\mathrm{a}, \mathrm{c}}$, Hiroko Inoue ${ }^{\mathrm{b}, \mathrm{c}}$, Yuhei Shiga ${ }^{\mathrm{a}, \mathrm{c}}$, Yohei Tsukihashi ${ }^{\mathrm{a}, \mathrm{b}}$, Tomoki Imaizumi ${ }^{\mathrm{a}}$, \\ Kenji Norimatsu ${ }^{a}$, Yoshiaki Idemoto ${ }^{a}$, Takashi Kuwano, Makoto Sugihara, \\ Hiroaki Nishikawa $^{\text {b, Yousuke Katsuda }}{ }^{\text {, Shin-ichiro Miura }}{ }^{\mathrm{a}, \mathrm{b}, \mathrm{d}}$
}

\begin{abstract}
Background: Although a recent study in a Japanese cohort indicated that extremely high-density lipoprotein cholesterol (HDL-C, $\geq 90 \mathrm{mg}$ / dL) had an adverse effect on atherosclerotic cardiovascular disease mortality, we could not conclude that high levels of HDL-C were associated with the presence or severity of coronary artery disease (CAD).

Methods: We enrolled 1,016 patients who were clinically suspected to have CAD and who underwent coronary computed tomography angiography (CCTA). The number of significantly stenosed coronary vessels (vessel disease (VD), $\geq 50 \%$ coronary stenosis is diagnosed as CAD) and the Gensini score were quantified using CCTA, and the lipid profile was measured. The patients were divided into four groups according to the HDL-C level: $<40 \mathrm{mg} / \mathrm{dL}$ ( $\mathrm{n}=115$, low), $40-59 \mathrm{mg} /$ $\mathrm{dL}(\mathrm{n}=530$, normal $), 60-89 \mathrm{mg} / \mathrm{dL}(\mathrm{n}=335$, high $)$ and $\geq 90 \mathrm{mg} / \mathrm{dL}$ ( $\mathrm{n}=36$, very-high).
\end{abstract}

Results: The percentage (\%) of CAD in the low, normal, high and very-high groups was $69 \%, 55 \%, 42 \%$ and $25 \%$, respectively ( $\mathrm{P}$ for trend $<0.01)$. The Gensini score in the low, normal, high and veryhigh groups was $20 \pm 25,12 \pm 16,8 \pm 12$ and $4 \pm 6$, respectively ( $P$ for trend $<0.01)$. The very-high group showed the lowest triglyceride (TG) levels among the four groups. There were no significant differences in the level of low-density lipoprotein cholesterol or $\%$ use of statin among the four groups. Finally, the presence of CAD

Manuscript submitted October 6, 2020, accepted October 17, 2020

Published online November 3, 2020

aDepartment of Cardiology, Fukuoka University School of Medicine, Fukuoka, Japan

bDepartment of Cardiology, Fukuoka University Nishijin Hospital, Fukuoka, Japan

${ }^{\mathrm{c}}$ These authors contributed equally to this manuscript.

${ }^{\mathrm{d} C}$ Corresponding Author: Shin-ichiro Miura, Department of Cardiology, Fukuoka University School of Medicine, 7-45-1 Nanakuma, Jonan-ku, Fukuoka 814-0180, Japan. Email: miuras@cis.fukuoka-u.ac.jp

doi: https://doi.org/10.14740/jocmr4367 was independently associated with a low level of HDL-C, in addition to age, male, high systolic blood pressure and hemoglobin Alc, but not TG, by a multivariate logistic regression analysis.

Conclusions: High levels of HDL-C at the time of CCTA for screening were associated with a reduced presence and severity of CAD.

Keywords: High-density lipoprotein cholesterol; Coronary artery disease; Coronary computed tomography angiography; Gensini score

\section{Introduction}

Advanced age, male, smoking, hypertension, dyslipidemia, obesity, family history of juvenile cardiovascular diseases (CVDs) and diabetes mellitus are critical risk factors for coronary artery disease (CAD) [1].

According to several large-scale randomized clinical trials, statin treatment for the primary and secondary prevention of CVD prevents one-third of the onset and progression of CVD, but not the remaining two-thirds [2]. There are several possible explanations for the residual risk of CVD. One is a low level of high-density lipoprotein cholesterol (HDL-C). According to the Japan Atherosclerosis Society Guidelines for Prevention of Atherosclerotic Cardiovascular Diseases 2017, serum HDL-C levels should be maintained $\geq 40 \mathrm{mg} / \mathrm{dL}$ for the primary and secondary prevention of CVD [3]. On the other hand, there is no significant clinical evidence regarding whether higher levels of HDL-C would be better for primary and secondary prevention. Rare variant in scavenger receptor BI raises HDL-C and increases risk of coronary heart disease [4]. Low and veryhigh HDL-C levels were associated with a higher risk of CV mortality as well as non-CV mortality [5]. Recently, extremely high levels of HDL-C ( $\geq 90 \mathrm{mg} / \mathrm{dL}$ ) were significantly associated with an increased risk of atherosclerotic cardiovascular disease (ASCVD) mortality and an increased risk of CAD and ischemic stroke in a pooled analysis of Japanese cohorts [6]. However, the prognostic value of higher HDL-C levels is con- 
troversial.

We have been studying the Coronary Computed Tomography Angiography (FU-CCTA) Registry at Fukuoka University in Japan and identified coronary risk factors for the primary prevention of CAD [7-12]. In this registry, none of the patients were diagnosed with CAD until the time of CCTA because we used CCTA to screen for CAD. When patients had significant coronary stenosis as assessed by CCTA, they were initially diagnosed with CAD.

We hypothesized that the target HDL-C level for primary coronary prevention should be higher, and that a higher level is better. Therefore, we examined the association between the HDL-C level at the time of CCTA and the presence and severity of CAD as assessed by CCTA using the FU-CCTA Registry.

\section{Materials and Methods}

\section{Study subjects}

One thousand sixteen consecutive subjects (66 years, 49\% male) who were clinically suspected of having CAD or who had at least one cardiac risk factor (aging, gender male, hypertension (HTN), dyslipidemia (DL), diabetes mellitus (DM), smoking, obesity, family history of CAD and/or chronic kidney disease (CKD)) underwent CCTA. Suspected CAD is defined as having some chest symptoms and/or electrocardiogram (ECG) abnormalities. All subjects underwent CCTA between 2012 and 2019. Patients with creatinine $>2.0 \mathrm{mg} / \mathrm{dL}$ or contrast-induced allergy did not undergo CCTA. The patients were divided into four groups according to the HDL-C level: $<40 \mathrm{mg} / \mathrm{dL}(\mathrm{n}=115$, low $), 40-59 \mathrm{mg} / \mathrm{dL}(\mathrm{n}=530$, normal $)$, $60-89 \mathrm{mg} / \mathrm{dL}(\mathrm{n}=335$, high) and $\geq 90 \mathrm{mg} / \mathrm{dL}(\mathrm{n}=36$, veryhigh). This study was conducted in compliance with the ethical standards of the responsible institution on human subjects as well as with the Helsinki Declaration. The protocol in this study was approved by the ethics committee of Fukuoka University Hospital, and all subjects gave their written informed consent to participate.

\section{Evaluation of coronary stenosis using CCTA}

We evaluated coronary stenosis using CCTA as previously described [5-8]. Patients were assessed by 64-MDCT on an Aquilion 64 (TOSHIBA, Tokyo, Japan) or by 320-MDCT on an Aquilion ONE ViSION (TOSHIBA, Tokyo, Japan). Fifteen coronary artery segments were assessed in all patients. Narrowing of the normal contrast-enhanced lumen to $\geq 50 \%$ that could be identified in multiplanar reconstructions or cross-sectional images was defined as significant stenosis. When patients had significant coronary stenosis, they were initially diagnosed with CAD. In addition, in all patients, the severity of CAD was assessed in terms of the number of significantly stenosed coronary vessels (VD) and the Gensini score [13]. Coronary artery calcification (CAC) was defined on CT images as the presence of more than two contiguous pixels with greater than 130 Hounsfield Units. The CAC score in each lesion was then computed by the Agatston method [14].

\section{Evaluation of risk factors for CAD}

Body mass index (BMI), systolic blood pressure (SBP), diastolic blood pressure (DBP), serum levels of triglyceride (TG), high-density lipoprotein cholesterol (HDL-C), lowdensity lipoprotein cholesterol (LDL-C), fasting blood sugar (FBS), hemoglobin A1c (HbAlc), smoking status and family history (myocardial infarction, angina pectoris or sudden death) were collected as risk factors in all patients. Information on medication use was obtained from medical records and included statin, fibrate, ezetimibe, eicosapentaenoic acid (EPA), biguanide, dipeptidyl peptidase-4 inhibitor (DPP-4I), insulin, angiotensin II receptor blocker/angiotensin-converting-enzyme inhibitor (ACEI/ARB), calcium channel blocker (CCB), $\beta$-blocker and diuretics. All of the blood samples were drawn in the morning after the patient had fasted overnight. The characteristics of patients were obtained from medical records with regard to history of hypertension (HTN), dyslipidemia (DL), diabetes mellitus (DM), CKD, smoking and family history of CAD.

\section{Statistical analysis}

A statistical analysis was performed using Excel 2016 (SSRI, Tokyo, Japan) and the Stat View statistical software package (Stat View 5; SAS Institute Inc., Cary, NC, USA) Continuous variables are shown as the mean \pm standard deviation. Categorical and continuous variables were analyzed for trends among the groups by the Cochran-Armitage trend test and Jonckheere-Terpstra trend test, respectively. A multivariate analysis was performed by a logistic regression analysis for independent variables (age, male, BMI, SBP, DBP, TG, HDL-C, LDL-C and $\mathrm{HbA} 1 \mathrm{c}$ ) that were related to the presence or absence of CAD. A value of $\mathrm{P}<0.05$ was considered significant.

\section{Results}

\section{Patient characteristics in all patients and the low, normal,} high and very-high HDL-C groups

Table 1 shows patient characteristics in all patients and the low, normal, high and very-high HDL-C groups. The HDL-C levels in all patients and the low, normal, high, and very-high HDL-C groups were 56, 35, 50, 70 and $101 \mathrm{mg} / \mathrm{dL}$, respectively. The HDL-C level increased as \%male, BMI, \%smoking, $\%$ DL, TG levels, \%DM, \%HTN, \% biguanide, \%DPP-4I and $\%$ ARB/ACEI decreased ( $\mathrm{P}$ for trend $<0.01$ ). The very-high group showed the lowest TG levels among the four groups. There were no significant differences in the LDL-C level or \% use of statin among the four groups. 
Table 1. Patient Characteristics in All Patients and the Low, Normal, High and Very-High HDL-C Groups

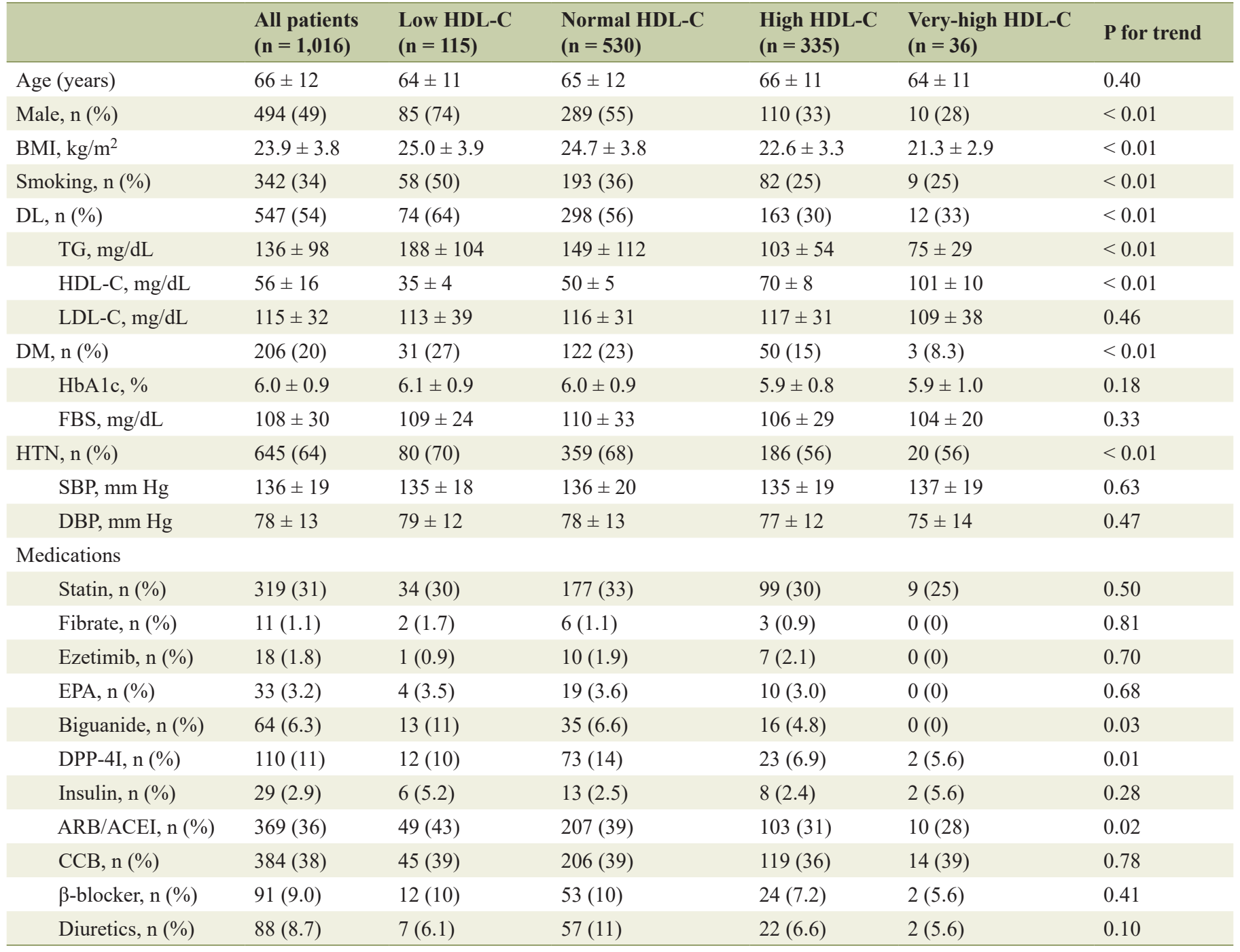

Continuous variables are expressed as mean \pm standard deviation. BMI: body mass index; DL: dyslipidemia; TG: triglyceride; HDL-C: high-density lipoprotein cholesterol; LDL-C: low-density lipoprotein cholesterol; DM: diabetes mellitus; HbA1c: hemoglobin A1c; FBS: fasting blood sugar; HTN: hypertension; SBP: systolic blood pressure; DBP: diastolic blood pressure; EPA: eicosapentaenoic acid; DPP-4I: dipeptidyl peptidase-4 inhibitor; ARB/ACEI: angiotensin II receptor blocker/angiotensin-converting-enzyme inhibitor; CCB: calcium channel blocker.

\section{Percentage of CAD, number of VD, Gensini score and CAC score in the low, normal, high and very-high HDL-C groups}

Percentage of CAD, number of VD, Gensini score and CAC score in the low, normal, high and very-high HDL-C groups are shown in Figure 1. Percentage of CAD in the low, normal, high and very-high groups was $69 \%, 55 \%, 42 \%$ and $25 \%$, respectively ( $\mathrm{P}$ for trend $<0.01$ ) (Fig. 1a). The number of VD was $1.5 \pm 1.2,1.0 \pm 1.1,0.7 \pm 1.0$ and $0.3 \pm 0.6$, respectively $(\mathrm{P}$ for trend $<0.01$ ) (Fig. 1b). The Gensini score was $20 \pm 25,12 \pm$ $16,8 \pm 12$ and $4 \pm 6$, respectively ( $\mathrm{P}$ for trend $<0.01$ ) (Fig. $1 \mathrm{c}$ ). There were no significant differences in the CAC score among the four groups (the CAC scores in the low, normal, high and very-high HDL-C groups were $280 \pm 671,220 \pm 558,218 \pm$ 665 and $62 \pm 162$, respectively).

\section{Independent predictors of CAD}

Table 2 shows independent predictors of CAD. The presence of CAD was independently associated with low levels of HDL-C, in addition to age, male, high SBP and HbA1c, but not with the levels of LDL-C or TG, by a multivariate logistic regression analysis.

\section{Discussion}

In this study, we hypothesized that higher HDL-C levels would be better for primary coronary prevention. We showed that, as the HDL-C level increased, \%CAD, number of VD and Gensini score decreased ( $\mathrm{P}$ for trend $<0.01)$. These results indicated 

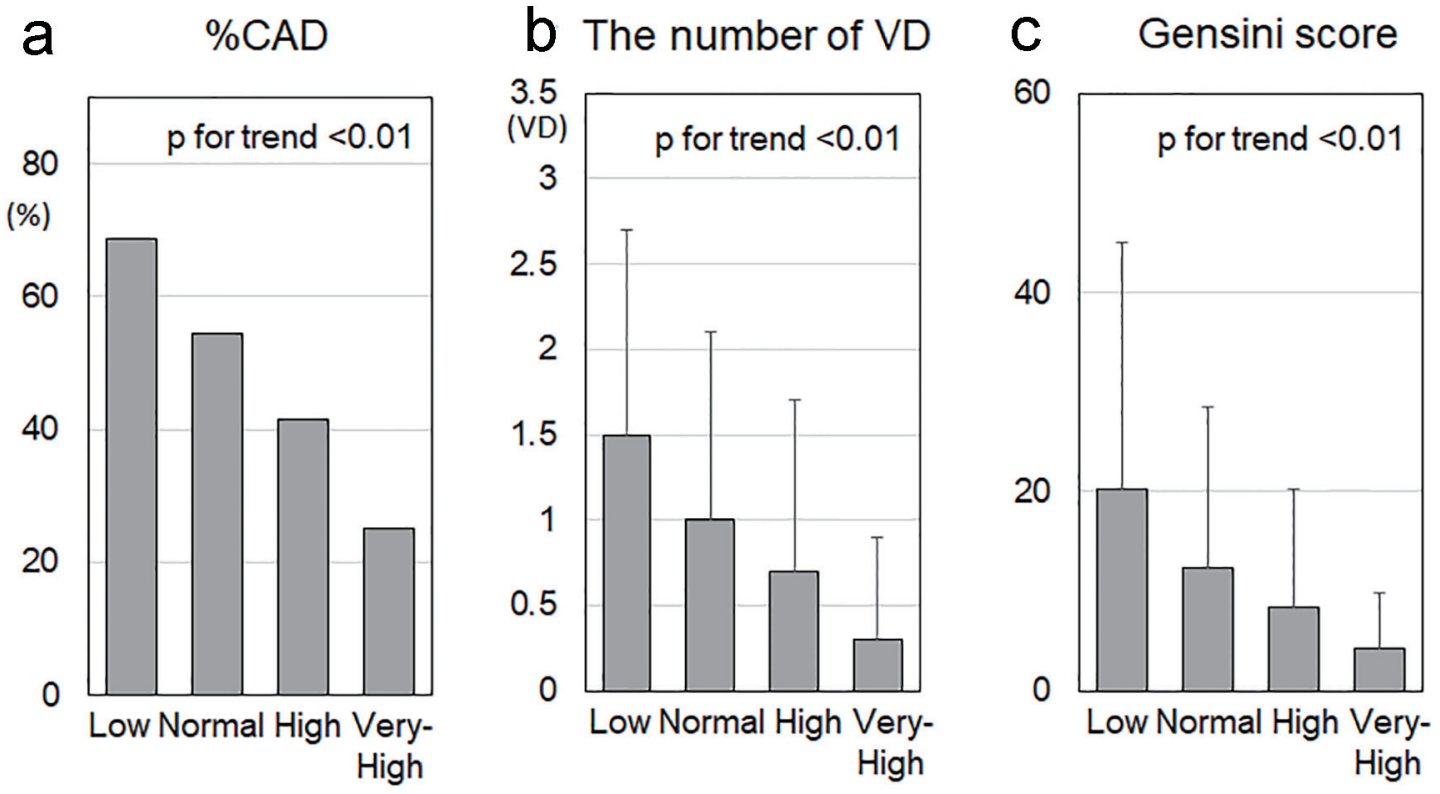

Figure 1. \%CAD (a), number of VD (b) and Gensini score (c) in the low, normal, high and very-high HDL-C groups. CAD: coronary artery disease; VD: vessel disease; HDL-C: high-density lipoprotein cholesterol. ${ }^{\dagger} P<0.05,{ }^{*} P<0.01,{ }^{* *} P<0.001,{ }^{* * *} P<$ 0.0001 .

that a higher HDL-C level at the time of CCTA for screening is better for preventing CAD.

The most important finding in this study was that the HDL-C level increased as \%CAD, number of VD and Gensini score decreased. The very-high HDL-C group (HDL-C $\geq 90$ $\mathrm{mg} / \mathrm{dL}$ ) showed the lowest \%CAD, number of VD and Gensini score among the four groups. In a ward, patients have less coronary atherosclerosis when they show higher levels of HDL-C. On the other hand, high HDL-C $(60-79 \mathrm{mg} / \mathrm{dL})$ levels have been shown to protect against $\mathrm{CAD}$, but higher levels had no additional benefit [15]. In addition, extremely high levels of HDL-C $(\geq 90 \mathrm{mg} / \mathrm{dL})$ had an adverse effect on ASCVD mortality in a pooled analysis of Japanese cohorts [6]. HDL mainly enhances reverse cholesterol transport, in which HDL takes up

Table 2. Independent Predictors of CAD

\begin{tabular}{llll}
\hline & OR & $\mathbf{9 5 \%}$ CI & P value \\
\hline Age & 1.05 & $1.04-1.07$ & $<0.0001$ \\
Male & 2.06 & $1.52-2.81$ & $<0.0001$ \\
BMI & 0.99 & $0.95-1.03$ & 0.73 \\
SBP & 1.02 & $1.01-1.03$ & $<0.01$ \\
DBP & 1.00 & $0.99-1.01$ & 0.87 \\
TG & 1.00 & $1.00-1.00$ & 0.60 \\
HDL-C & 0.98 & $0.97-0.99$ & $<0.0001$ \\
LDL-C & 1.00 & $0.99-1.00$ & 0.58 \\
HbA1c & 1.32 & $1.12-1.57$ & $<0.01$ \\
\hline
\end{tabular}

OR: odds ratio; $\mathrm{Cl}$ : confidence interval; BMI: body mass index; SBP: systolic blood pressure; DBP: diastolic blood pressure; TG: triglyceride; HDL-C: high-density lipoprotein cholesterol; LDL-C: low-density lipoprotein cholesterol; HbA1c: hemoglobin A1c. peripheral cholesterol and transfers it to the liver for excretion in the bile and feces. Cholesteryl ester transfer protein (CETP) inhibitors drastically increase HDL-C levels in blood, whereas CETP inhibition provides insufficient cardiovascular benefit $[16,17]$. We do not consider these previous reports to be inconsistent with our present findings, since these previous reports looked at the prognosis and we looked at the relevance of when patients were initially diagnosed CAD at the time of CCTA. We think that the HDL-C level is significantly associated with the severity of coronary atherosclerosis at the time of primary prevention when patients are initially diagnosed with CAD. After various therapies, the association may gradually become weaker for secondary prevention. In addition, the cut-off level of HDL-C that gave the greatest sensitivity and specificity for the diagnosis of CAD was $55 \mathrm{mg} / \mathrm{dL}$. This is not a high level of HDL-C.

Another important issue is HDL functionality, which is involved in cholesterol efflux capacity, as well as its anti-oxidative, anti-inflammatory, anti-proliferative and antithrombotic effects $[18,19]$. Cholesterol efflux capacity was inversely associated with the incidence of cardiovascular events, restenosis rates after coronary stent implantation and graft failure in renal transplant recipients [20-22]. Thus, we should consider not only the level of HDL-C but also HDL functionality. We previously reported that the calculated total cholesterol efflux capacity that depended on the HDL-C level was significantly correlated with the presence of $\mathrm{CAD}$ at the time of CCTA [23].

The presence of CAD was independently associated with a low level of HDL-C, in addition to age, male, high SBP and HbA1c, but not LDL-C or TG. Age, male, HTN and DM are all critical coronary risk factors [1]. SBP is a more critical factor than DBP with regard to the onset of CAD [1]. It is reason- 
able that the presence of CAD was independently associated with high SBP. Although elevated levels of LDL-C and TG are coronary risk factors, average levels of LDL-C and TG in this study were 115 and $136 \mathrm{mg} / \mathrm{dL}$, respectively. Target levels of LDL-C and TG for the primary prevention of ASCVD are < 120 and $<150 \mathrm{mg} / \mathrm{dL}$ in patients at high risk, respectively [3]. Therefore, the presence of CAD may not be independently associated with LDL-C and TG levels.

This study has several important limitations. First, this study was cross-sectional. Second, the actual number of subjects having CAD in very-high HDL-C group was very low because the number of patients in the very-high group was small. Third, CCTA is not a gold standard for the evaluation of $\mathrm{CAD}$, although recent studies have shown that its sensitivity and specificity were both approximately $95 \%$ of those for invasive coronary angiography for the identification of significant coronary stenosis [24]. A large-scale prospective study will be needed to address these issues.

In conclusion, high levels of HDL-C at the time of CCTA for screening were associated with a reduced presence and severity of CAD.

\section{Acknowledgments}

We thank all of the members of the Department of Cardiology, Fukuoka University Hospital, and Department of Cardiology, Fukuoka University Nishijin Hospital, Fukuoka, Japan.

\section{Financial Disclosure}

None to declare.

\section{Conflict of Interest}

We have no conflict of interest.

\section{Informed Consent}

Informed consent was obtained.

\section{Author Contributions}

Conceptualization: KT, SM. Validation: HI, YS, TI, YT. Formal analysis: KN, YI, TK. Investigation: KT, HI, HN, YK, YT. Data curation: HI, YS, YK. Writing original draft: YS, MS. Writing review and editing: SM. Supervision: SM.

\section{Data Availability}

The authors declare that data supporting the findings of this study are available within the article.

\section{References}

1. Umemura S, Arima H, Arima S, Asayama K, Dohi Y, Hirooka Y, Horio T, et al. The Japanese Society of Hypertension Guidelines for the management of hypertension (JSH 2019). Hypertens Res. 2019;42(9):1235-1481.

2. Chapman MJ, Redfern JS, McGovern ME, Giral P. Niacin and fibrates in atherogenic dyslipidemia: pharmacotherapy to reduce cardiovascular risk. Pharmacol Ther. 2010;126(3):314-345.

3. Kinoshita $\mathrm{M}$, Yokote $\mathrm{K}$, Arai $\mathrm{H}$, Iida M, Ishigaki Y, Ishibashi S, Umemoto S, et al. Japan Atherosclerosis Society (JAS) guidelines for prevention of atherosclerotic cardiovascular diseases 2017. J Atheroscler Thromb. 2018;25(9):846-984.

4. Zanoni P, Khetarpal SA, Larach DB, Hancock-Cerutti WF, Millar JS, Cuchel M, DerOhannessian S, et al. Rare variant in scavenger receptor BI raises HDL cholesterol and increases risk of coronary heart disease. Science. 2016;351(6278):1166-1171.

5. Ko DT, Alter DA, Guo H, Koh M, Lau G, Austin PC, Booth GL, et al. High-density lipoprotein cholesterol and cause-specific mortality in individuals without previous cardiovascular conditions: the CANHEART study. J Am Coll Cardiol. 2016;68(19):2073-2083.

6. Hirata A, Sugiyama D, Watanabe M, Tamakoshi A, Iso H, Kotani K, Kiyama M, et al. Association of extremely high levels of high-density lipoprotein cholesterol with cardiovascular mortality in a pooled analysis of 9 cohort studies including 43,407 individuals: The EPOCH-JAPAN study. J Clin Lipidol. 2018;12(3):674-684 e675.

7. Mitsutake R, Miura S, Kawamura A, Saku K. Are metabolic factors associated with coronary artery stenosis on MDCT? Circ J. 2009;73(1):132-138.

8. Mitsutake R, Miura S, Zhang B, Saku K. HDL-associated factors provide additional prognostic information for coronary artery disease as determined by multi-detector row computed tomography. Int J Cardiol. 2010;143(1):72-78.

9. Mitsutake R, Miura S, Shiga Y, Uehara Y, Saku K. Association between hypertension and coronary artery disease as assessed by coronary computed tomography. J Clin Hypertens (Greenwich). 2011;13(3):198-204.

10. Shiga Y, Miura S, Mitsutake R, Kawamura A, Uehara Y, Saku K. Significance of serum high-density lipoprotein cholesterol levels for diagnosis of coronary stenosis as determined by MDCT in patients with suspected coronary artery disease. J Atheroscler Thromb. 2010;17(8):870878.

11. Norimatsu K, Miura S, Suematsu Y, Shiga Y, Miyase Y, Nakamura A, Yamada M, et al. Associations between glycated albumin or hemoglobin A1c and the presence of coronary artery disease. J Cardiol. 2015;65(6):487-493.

12. Ueda Y, Shiga Y, Idemoto Y, Tashiro K, Motozato K, Koyoshi R, Kuwano T, et al. Association between the presence or severity of coronary artery disease and pericardial fat, paracardial fat, epicardial fat, visceral fat, and subcutaneous fat as assessed by multi-detector row computed tomography. Int Heart J. 2018;59(4):695-704. 
13. Gensini GG. A more meaningful scoring system for determining the severity of coronary heart disease. Am J Cardiol. 1983;51(3):606.

14. Agatston AS, Janowitz WR, Hildner FJ, Zusmer NR, Viamonte M, Jr., Detrano R. Quantification of coronary artery calcium using ultrafast computed tomography. J Am Coll Cardiol. 1990;15(4):827-832.

15. Hirata A, Okamura T, Sugiyama D, Kuwabara K, Kadota A, Fujiyoshi A, Miura K, et al. The relationship between very high levels of serum high-density lipoprotein cholesterol and cause-specific mortality in a 20-year followup study of Japanese general population. J Atheroscler Thromb. 2016;23(7):800-809.

16. Di Bartolo BA, Psaltis PJ, Bursill CA, Nicholls SJ. Translating Evidence of HDL and Plaque Regression. Arterioscler Thromb Vasc Biol. 2018;38(9):1961-1968.

17. Armitage J, Holmes MV, Preiss D. Cholesteryl ester transfer protein inhibition for preventing cardiovascular events: JACC review topic of the week. J Am Coll Cardiol. 2019;73(4):477-487.

18. Farbstein D, Levy AP. HDL dysfunction in diabetes: causes and possible treatments. Expert Rev Cardiovasc Ther. 2012;10(3):353-361.

19. Sviridov D, Mukhamedova N, Remaley AT, Chin-Dusting J, Nestel P. Antiatherogenic functionality of high density lipoprotein: how much versus how good. J Atheroscler Thromb. 2008;15(2):52-62.

20. Rohatgi A, Khera A, Berry JD, Givens EG, Ayers CR, Wedin KE, Neeland IJ, et al. HDL cholesterol efflux capacity and incident cardiovascular events. N Engl J Med. 2014;371(25):2383-2393.

21. Imaizumi S, Miura S, Takata K, Takamiya Y, Kuwano T, Sugihara M, Ike A, et al. Association between cholesterol efflux capacity and coronary restenosis after successful stent implantation. Heart Vessels. 2016;31(8):1257-1265.

22. Annema W, Dikkers A, de Boer JF, Dullaart RP, Sanders JS, Bakker SJ, Tietge UJ. HDL cholesterol efflux predicts graft failure in renal transplant recipients. J Am Soc Nephrol. 2016;27(2):595-603.

23. Norimatsu K, Kuwano T, Miura SI, Shimizu T, Shiga Y, Suematsu Y, Miyase Y, et al. Significance of the percentage of cholesterol efflux capacity and total cholesterol efflux capacity in patients with or without coronary artery disease. Heart Vessels. 2017;32(1):30-38.

24. Ropers D, Rixe J, Anders K, Kuttner A, Baum U, Bautz W, Daniel WG, et al. Usefulness of multidetector row spiral computed tomography with 64- x 0.6-mm collimation and 330-ms rotation for the noninvasive detection of significant coronary artery stenoses. Am J Cardiol. 2006;97(3):343-348. 